'Departamento de Anatomía Patológica. Facultad de Medicina. Universidad de La Frontera. Temuco, Chile. 2 Scentifical and Technological Bioresources Nucleus (BIOREN) Servicio de Radiología Hospital Hernán Henríquez Aravena; Temuco.

Recibido el 28 de febrero de 2011, aceptado el 14 octubre de 2011

Correspondencia a: Dr. Oscar Tapia E. Departamento de Anatomía Patológica, Facultad de Medicina, Universidad de La Frontera. Manuel Montt 112 Fono: 045-325735. Código Postal 478-1176. Temuco, Chile. E-mail: otescalona@gmail.

\section{Hidatidosis cerebral: aspectos clínicos e imagenológicos. Reporte de 4 casos}

\author{
OSCAR TAPIA E. ${ }^{1,2}$, AARÓN VIDAL T. ${ }^{3}$, LILIA ANTONIO P. ${ }^{1,2}$
}

\section{Brain hydatidosis. Report of four cases}

In only $2 \%$ of all cases of hydatidosis, the cysts are located in the brain. We report a 12-year-old male, a 5-year-old girl and a 19-year-old female consulting for intracranial hypertension and a 13-year-old male consulting for a left hemiparesis. Imaging studies found intra-cerebral cysts with characteristics of hydatidosis in all. All lesions were completely removed surgically and the pathological study of the excised piece confirmed the diagnosis of hydatidosis.

(Rev Med Chile 2012; 140: 358-363).

Key words: Brain neoplasms; Cysts; Echinococcosis, cerebral.
L a hidatidosis es la zoonosis parasitaria más frecuente en Chile. La tasa de incidencia nacional notificada en el año 2004 fue de 2,1 x 100.000 habitantes, predominando en las áreas ganaderas, especialmente de la zona sur y austral de nuestro país. Es causada por la forma larval del céstodo Echinococcus granulosus, donde el perro es el hospedero definitivo y el ganado ovino y bovino, los hospederos intermediarios. El hombre es un hospedero intermediario accidental por consumo de alimentos o agua contaminada con huevos presentes en las heces de los animales ${ }^{1-5}$.

El E. granulosus puede alcanzar cualquier órgano o tejido, formando lesiones quísticas únicas o múltiples, siendo el hígado (59-75\%) y pulmón (8-43\%) los órganos más frecuentemente afectados $^{6-8}$. Otros órganos, menos frecuentemente comprometidos, son bazo, riñón, páncreas, mama, peritoneo, hueso y tejidos blandos, tiroides, corazón, médula espinal y encéfalo. El compromiso encefálico corresponde a 1-2\% de los casos y representa $2-4 \%$ de las lesiones intracraneales no traumáticas, afectando más frecuentemente a pacientes pediátricos en 50-75\% de los $\operatorname{casos}^{6,9-12}$.

A pesar de ser una infestación con alta prevalencia en nuestro país, el compromiso encefálico representa una localización inusual con escasos reportes a nivel nacional ${ }^{13,14}$. Por esta razón, presentamos cuatro casos de pacientes con hidatidosis cerebral diagnosticados entre noviembre de 2009 y agosto de 2010 en el Hospital Hernán Henríquez Aravena de Temuco, discutiéndose sus principales características clínicas e imagenológicas.

\section{Caso 1}

Escolar de 12 años, sexo masculino con residencia rural en la IX región. Consultó por cuadro de 6 meses de evolución caracterizado por manifestaciones clínicas de tipo hipertensión intracraneana (cefalea holocránea recurrente [2-3 veces por semana], mareos y vómitos ocasionales) e irritativa (dos episodios de crisis convulsivas tónico-clónicas generalizadas). El electroencefalograma (EEG) fue normal y se realizó una tomografía computada (TC) cerebral que mostró un quiste intraxial temporal izquierdo de $65 \mathrm{~mm}$ de diámetro, bien delimitado y con severo efecto de masa, desviación de línea media de $8 \mathrm{~mm}$, herniación uncal e hidrocefalia (Figura 1A). La resonancia magnética (RM) mostró además un doble contorno en la periferia, por desprendimiento de la membrana germinal, con una ligera impregnación tras la administración de gadolinio (Figura 1B y C).

El examen de fondo de ojo demostró edema de papila bilateral, sin evidenciarse déficit motor ni 

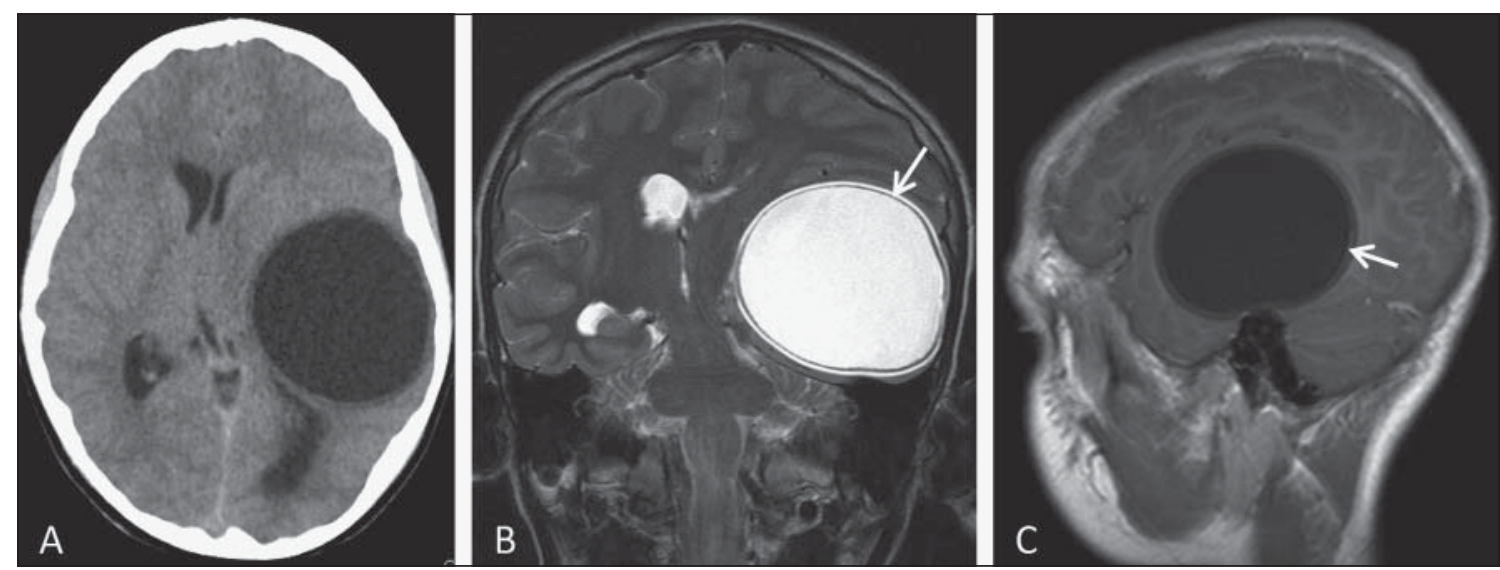

Figura 1. TC axial sin contraste (A). RM T2WI coronal (B). RM T1WI sagital con gadolinio (C). Formación quística intraaxial temporal izquierda, bien delimitada y de contenido homogéneo isodenso (A) e isointenso (B, C) respecto al LCR. Significativo efecto de masa con hidrocefalia secundaria (A). Membrana germinal (B y C, flecha) separada del resto del quiste y que presenta una discreta captación de contraste (C).

sensitivo al examen físico. El hemograma y pruebas hepáticas resultaron normales, sin demostrarse la presencia de lesiones quísticas en la radiografía (Rx) de tórax ni en la ecotomografía abdominal. Se planteó el diagnóstico de hidatidosis cerebral y se realizó la exéresis completa e intacta del quiste, confirmando el estudio histopatológico la etiología hidatídica de la lesión (Figura 2).

El paciente evolucionó favorablemente, asintomático, sin déficit neurológico ni complicaciones post operatorias.

\section{Caso 2}

Preescolar de 5 años, sexo femenino con residencia urbana en la IX región. Consultó por historia de 4 meses de evolución de déficit motor progresivo y manifestaciones clínicas de hipertensión intracraneana en el último mes (cefalea y vómitos). El examen clínico demostró hemiparesia braquio-crural derecha con reflejo patelar y aquiliano exaltados, además de midriasis izquierda. Se solicitó TC cerebral que mostró un gran quiste

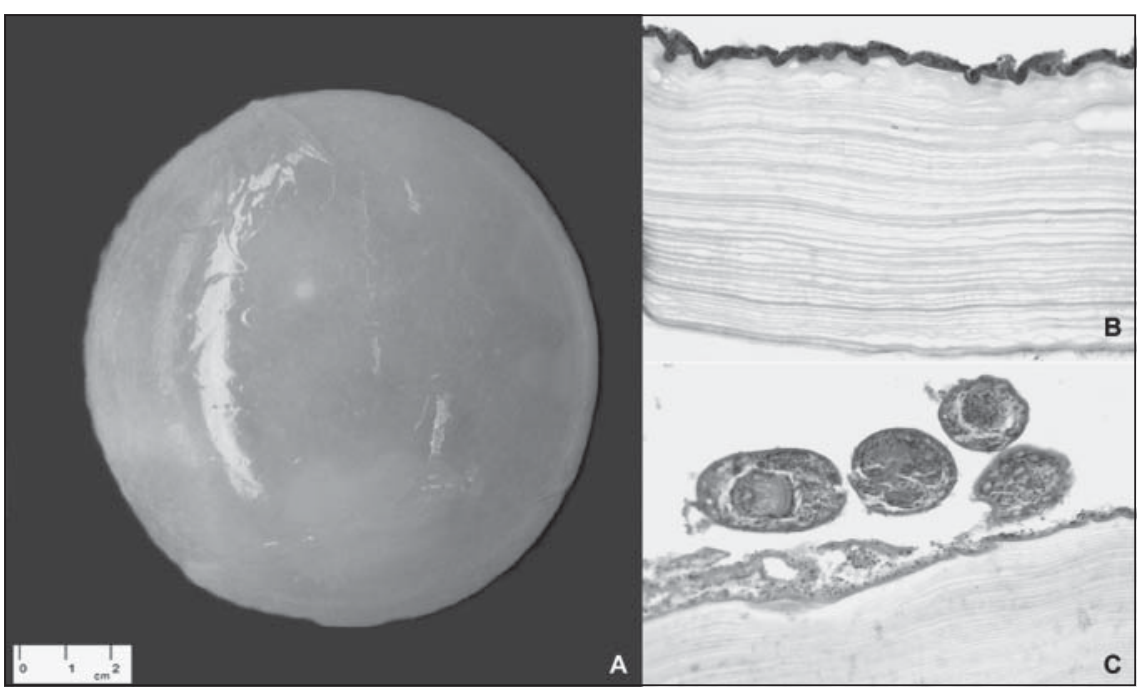

Figura 2. A: Lesión quística unilocular, translúcida, de bordes bien definidos y contenido líquido de aspecto "agua de roca". B: (HE, 200x) y C (HE, 100x). Estructuras laminares basófilas, acelulares de disposición paralela (membranas hidatídicas) con escólices en el interior de la cavidad quística (C). 


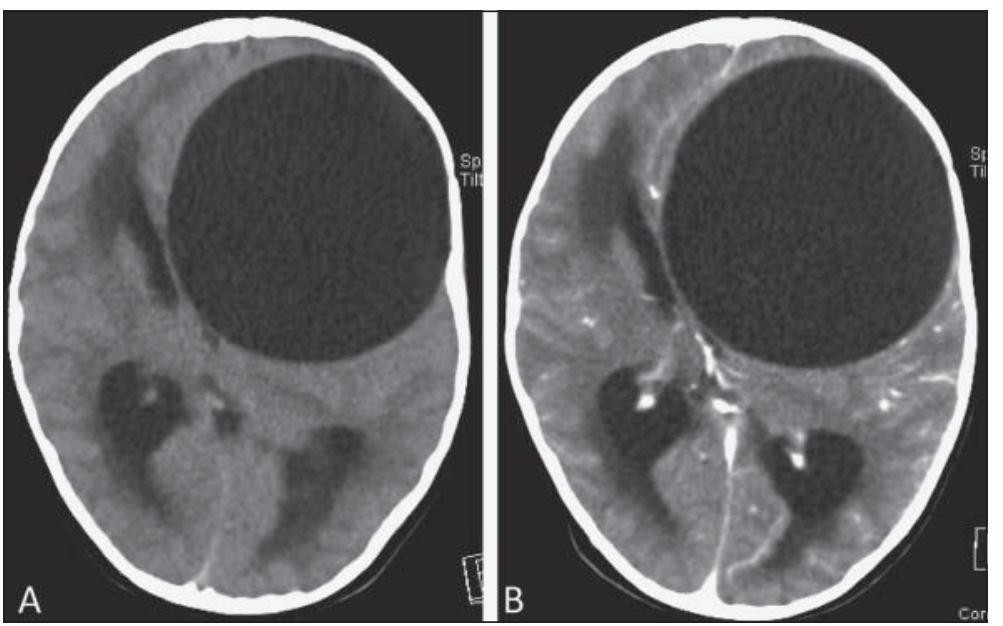

Figura 3. TC axial sin contraste (A) y con contraste (B). Formación quística intraaxial frontal izquierda, sin edema perilesional y con contenido isodenso respecto al LCR (A) y que no presenta captación de contraste (B). Severo efecto de masa con desviación de línea media e hidrocefalia secundaria. intraxial frontal izquierdo de $90 \mathrm{~mm}$ de diámetro, bien definido, homogéneo, sin edema perilesional ni captación de contraste. Severo efecto de masa con desviación de línea media de aproximadamente $20 \mathrm{~mm}$ y dilatación de los ventrículos laterales con signos de edema transependimario (Figura 3).

El hemograma y pruebas hepáticas fueron normales, sin demostrarse la presencia de lesiones quísticas en la Rx de tórax ni en la ecotomografía abdominal. Se planteó el diagnóstico de quiste hidatídico frontal izquierdo y se realizó la exéresis intacta del quiste. El estudio histopatológico confirmó la etiología hidatídica de la lesión.

El paciente evolucionó favorablemente, con recuperación completa de la movilidad del hemicuerpo derecho.

\section{Caso 3}

Paciente de sexo femenino de 19 años de edad con residencia rural en la IX región, sin antecedentes mórbidos. Consultó por cuadro de 5 meses de evolución de sintomatología secundaria a síndrome de hipertensión intracraneana (cefalea recurrente y progresiva asociada a vómitos, refractaria a tratamiento médico y edema papilar bilateral acentuado al fondo de ojo). La TC cerebral y RM mostraron una gran formación quística intraxial temporal izquierda, de $60 \mathrm{~mm}$ de diámetro de características similares a los casos anteriores y con moderado efecto de masa sobre el parénquima adyacente y sistema ventricular, con desviación de línea media de 5,5 mm (Figura 4).

El estudio con $\mathrm{Rx}$ de tórax y ecotomografía abdominal no mostró lesiones quísticas. El hemograma no mostró eosinofilia y las pruebas hepáticas fueron normales. Se planteó el diagnóstico de quiste hidatídico temporal izquierdo. Se realizó la exéresis intacta de la lesión, confirmando el estudio histopatológico la etiología hidatídica.

La paciente evolucionó favorablemente, sin déficit neurológico ni complicaciones post operatorias.

\section{Caso 4}

Escolar de 13 años, sexo masculino con residencia rural en la IX región, sin antecedentes mórbidos. Consultó por presentar déficit motot progresivo (hemiparesia braquio-crural izquierda) y cefalea progresiva. La TC cerebral mostró un quiste intraxial temporal derecho de $60 \mathrm{~mm}$ de diámetro, bien delimitado, isodenso respecto al LCR, sin captación de contraste. Significativo efecto de masa con desviación de línea media de $7 \mathrm{~mm}$ y herniación uncal (Figura 5). Se planteó el diagnóstico de quiste hidatídico temporal derecho; se realizó la exéresis intacta del quiste y el estudio anatomopatológico confirmó la etiología hidatídica. El estudio con Rx de tórax y ecotomografía abdominal no mostró lesiones quísticas.

El paciente evolucionó favorablemente, sin complicaciones quirúrgicas ni déficit neurológico. 

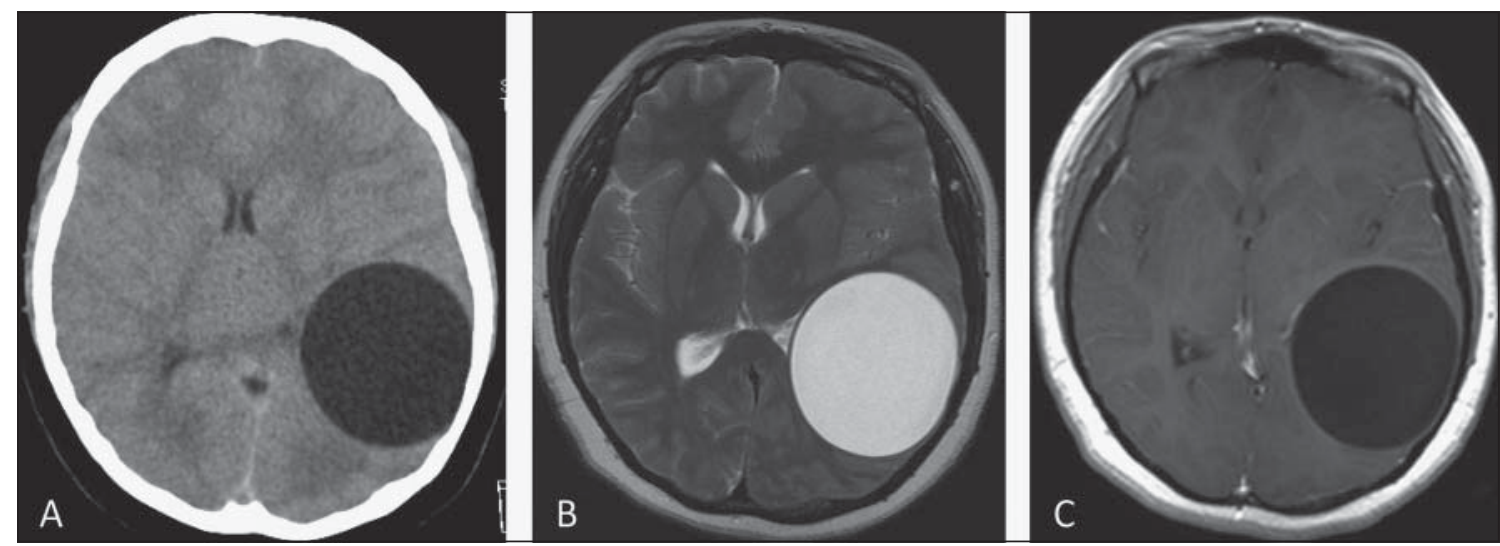

Figura 4. TC axial sin contraste (A). RM T2WI axial (B). RM T1WI axial con gadolinio (C). Formación quística intraaxial temporal izquierda de contornos regulares y bien definidos, sin edema perilesional, con contenido homogéneo isodenso ( $A$ ) e isointenso ( $B$, C) respecto al LCR. Moderado efecto de masa sobre el parénquima y sistema ventricular. No se aprecia captación de contraste (C).
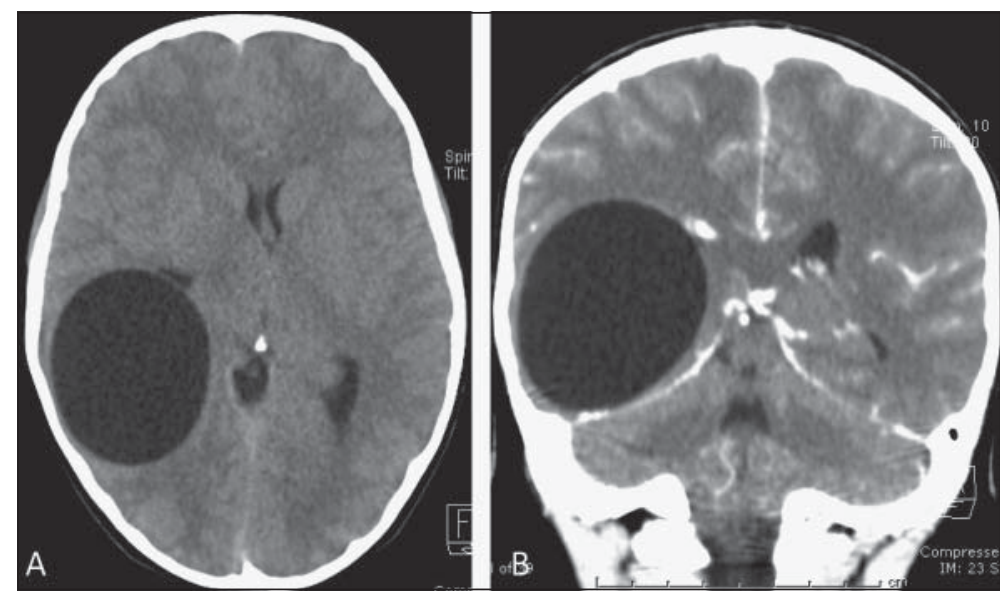

Figura 5. TC axial sin contraste (A) y con contraste (B). Formación quística intraaxial temporal derecha, sin edema perilesional, con contenido homogéneo isodenso respecto al LCR (A) y sin captación de contraste (B). Moderado efecto de masa sobre las estructuras adyacentes.

\section{Discusión}

La hidatidosis o equinococcosis es la infección causada por la forma larval del céstodo E. granulosus y con menos frecuencia E. multilocularis. Esta infección presenta una distribución mundial, siendo en Chile la zoonosis parasitaria más frecuente y representando un problema de salud pública especialmente en la zona sur y austral de nuestro país, hecho que ha motivado amplias campañas de educación sanitaria destinadas al control y prevención de la enfermedad ${ }^{1-5}$.

El compromiso encefálico por E. granulosus representa sólo el 1-2\% de los casos de hidatidosis y afecta fundamentalmente a niños y adultos jóve- nes $(60-93 \% \text { de los casos })^{10-12,14}$. Los cuatro casos presentados corresponden a este grupo etario. Si bien algunos autores reportan que los niños varones son mayormente afectados ${ }^{11,12,15}$, otros como Limaiem et al. ${ }^{16}$, no encuentran diferencias significativas.

Los hallazgos desde el punto de vista imagenológico son característicos, tratándose generalmente de lesiones quísticas únicas, habitualmente uniloculares y menos frecuentemente multiloculares, de localización principalmente intraaxial y con mayor frecuencia cerebrales hemisféricas en el territorio vascular de la arteria cerebral media debido a la llegada por vía hematógena del parásito. La lesión habitualmente es bien definida, de contenido 
isodenso e isointenso respecto al LCR, sin edema perilesional y mínima o ausente captación de contraste en su periferia ${ }^{11,17-20}$. Los cuatro casos reportados presentaron lesiones quísticas únicas, uniloculares cerebrales hemisféricas, tres en el lóbulo temporal y una frontal, presentando uno de ellos mínima impreganación de gadolinio en la RM. En ocasiones se puede visualizar la membrana germinal desprendida del resto de la estructura del quiste ${ }^{17,18}$, tal como en el caso 1 (Figura 1B) y en forma excepcional $(<1 \%)$ presencia de calcificaciones ${ }^{11}$. El efecto de masa ejercido por estas lesiones sobre las diferentes estructuras encefálicas es el principal responsable de las manifestaciones clínicas de la enfermedad, las cuales variarán de acuerdo a la localización del quiste. Bükte et al. ${ }^{11}$ reportaron cefalea (83\%), edema de papila (77\%), vómitos $(66 \%)$, hemiparesia (38\%), convulsiones (33\%), alteraciones visuales (27\%), ataxia (11\%) y parálisis facial (5\%). Respecto a la sintomatología neurológica, todos nuestros casos presentaron síntomas/signos secundarios a hipertensión intracraneana (cefalea, vómitos y/o edema de papila). Se observó convulsiones en un caso $(25 \%)$ y déficit motor en dos (50\%), revirtiendo los signos y síntomas una vez realizada la cirugía.

El efecto de masa de la lesión determina algunas de las principales complicaciones, como hidrocefalia obstructiva, desviación de línea media y herniaciones ${ }^{11,17,18,21}$. Tres de los casos reportados aquí presentaron estas complicaciones.

Desde el punto de vista imagenológico, el diagnóstico diferencial es variado y comprende lesiones quísticas tumorales (astrocitoma quístico), infecciosas (abscesos, neurocisticercosis) y congénitas (quiste aracnoidal, quiste epidermoide, quiste porencefálico). El estudio imágenológico, TC y principalmente RM es fundamental en excluir las otras causas. Algunas características específicas de la lesión, asociadas a la indemnidad del parénquima adyacente, sin existir compromiso infiltrativo, edema, encefalomalacia o gliosis, y sumadas a la ausencia de algunos elementos como heterogenidad o irregularidad de la lesión, componentes sólidos, restricción de la difusión y la mínima o ausente captación de contraste permiten, en su conjunto, realizar un adecuado diagnóstico. En los cuatro casos presentados el diagnóstico imagenológico prequirúrgico fue hidatidosis encefálica, no contando en nuestra experiencia con diagnósticos radiológicos falsamente positivos; debiendo mencionar que estos examenes (TC y RM) son evaluados en su totalidad por neurorradiólogos. Por su parte, la espectroscopia por RM no tiene un rol relevante en el diagnóstico, sólo en el algunos casos infrecuentes de hidatidosis encefálica con lesiones complejas podría aportar algunos elementos diferenciadores, sin ser de todas formas fundamental para este.

El tratamiento de elección consiste en la exéresis intacta de la lesión, la cual se logra hasta en el $70 \%$ de los casos. En los cuatro casos presentados se efectuó la exéresis completa e intacta de la lesión mediante la técnica de Dowling-Orlando que consiste en la realización de una craneotomía amplia con posterior apertura de la adventicia e irrigación de solución salina entre el parénquima cerebral y el quiste que permita la salida intacta de la prolígera y su contenido por gravedad, luego inclinar la cabeza del paciente (parto del quiste). La recurrencia secundaria a la ruptura intra-operatoria en tanto alcanza el 40,7\%9, 16,22-24.

En nuestros pacientes sólo se efectuó tratamiento quirúrgico, sin presentar recurrencia o quistes en otras localizaciones al momento de este reporte y luego de transcurrido 19, 16, 14 y 11 meses respectivamente. Por otro lado, la indicación de tratamiento farmacológico con antiparasitarios benzoimidazólicos (mebendazol y albendazol) estaría recomendado para casos inoperables por compromiso de estructuras vitales o multifocales, ruptura intra-operatoria del quiste y recurrencia; teniendo en consideración que el tamaño y "edad" del quiste son de los factores determinantes más importantes en la respuesta al tratamiento, así lesiones pequeñas y de corta evolución presentan paredes más delgadas que permitirían una mejor penetración y respuesta al fármaco ${ }^{14,25-27}$.

Los reportes indican que entre 6\% y $63 \%$ de estos pacientes presentan enfermedad hidatídica en otras localizaciones ${ }^{11,28,29}$, sin embargo, en una proporción importante de los casos la afección cerebral representa una verdadera localización primaria de la enfermedad, sin pesquisarse quistes en otras localizaciones; tal cual sucedió en nuestros pacientes.

La elevada incidencia de hidatidosis en nuestro país y muy especialmente en nuestra región, hacen que esta patología deba ser siempre considerada dentro de los diagnósticos diferenciales de lesiones quísticas encefálicas, cada una de ellas con implicancias terapéuticas y pronósticas muy diferentes. 


\section{Referencias}

1. Sapunar J. Parasitología Médica. Santiago, Mediterráneo Ltda., 1999; 39: 338-54.

2. Serra I, Araya C, Araneda J. Evolución epidemiológica de la hidatidosis humana en Chile, 1965-1988. Impacto de los programas ganaderos de la XII y XI regiones. Rev Med Chile 1993; 121: 343-9.

3. Serra I, Araya C, Araneda J. Situación actual de la hidatidosis humana en Chile. Dos proposiciones de corrección de la subnotificación. Rev Med Chile 1995; 123: 659-69.

4. Serra I, Araneda J, Araya C. Análisis regional de la hidatidosis humana y animal en Chile, 1989-1993. Bol Chil Parasitol 1996; 51: 3-12.

5. Ministerio de Salud de Chile. Enfermedades de Notificación obligatoria. http://epi.minsal.cl/epi/html/ AtlasInteractivos/Nacionales/Atlas_Zoo_10/Hida.htm

6. Yuksel M, Demirpolat G, Sever A, Bakaris S, Bulbuloglu E, Elmas N. Hydatid disease involving some rare locations in the body: a pictorial essay. Korean J Radiol 2007; 8: 531-40.

7. Khiari A, Fabre JM, Mzali R, Domergue J, Beyrouti MI. Unusual locations of hydatid cysts. Ann Gastroenterol Hepatol 1995; 31: 295-305.

8. Yilmaz N, Kiymaz N, Etlik O, Yazici T. Primary hydatid cyst of the brain during pregnancy. Neurol Med Chir 2006; 46: 415-7.

9. Ciurea AV, Fountas KN, Coman TC, Machinis TG, Kapsalaki EZ, Fezoulidis NI, et al. Long term surgical outcome in patients with intracranial hydatid cyst. Acta Neurochir 2006; 148: 421-6.

10. Onal C, Unal F, Barlas O, Izgi N, Hepgul K, Turantan MI, et al. Long-term follow-up and results of 30 pediatric intracranial hydatid cysts: half a century of experience in the department of neurosurgery of the school of Medicine at the university of Istanbul (1952-2001). Pediatr Neurosurg 2001; 35: 72-81.

11. Bükte Y, Kemaloglu S, Nazaroglu H, Ozkan U, Ceviz A, Simsek M. Cerebral hydatid disease: CT and MR imaging findings. Swiss Med Wkly 2004; 134: 459-67.

12. Lunardi P, Missori P, Di Lorenzo N, Fortuna A. Cerebral hydatidosis in childhood: a retrospective survey with emphasis on long-term follow-up. Neurosurgery 1991; 29: 515-8.

13. Stephens C; Guajardo U. Hydatidosis of the nervous system in Temuco IX region of Chile. Rev Méd Sur 1991; 16: 44-6.

14. Noemí I, Viovy A, Zamorano R, Blanco A, Revello D, Vojkovic M, et al. [Hydatid disease in childhood: Albendazole in its medical and surgical treatment]. Rev Chil Infect 2003; 20: 229-234.
15. Beskonakli E, Cayli S, Yalcinlar Y. Primary intracranial extradural hydatid cyst extending above and below the tentorium. Br J Neurosurg 1996; 10: 315-6.

16. Limaiem F, Bellil S, Bellil K, Chelly I, Mekni A, Khaldi M, et al. Primary hydatidosis of the central nervous system: a retrospective study of 39 Tunisian cases. Clin Neurol Neurosurg 2010; 112: 23-8.

17. Polat P, Kantarci M, Alper F, Suma S, Koruyucu M, Okur A. Hydatid disease from head to toe. Radiographics 2003; 23: 475-94.

18. Tlili-Graiess K, El-Ouni F, Gharbi-Jemni H, Arifa N, Moulahi H, Mrad-Dali K et al. Cerebral hydatid disease: imaging features. J Neuroradiol 2006; 33: 304-18.

19. Osborn AG, Preece MT. Intracranial cysts: radiologicpathologic correlation and imaging approach. Radiology. 2006; 239: 650-64.

20. Ellison D, Love S, Chimelli L, Harding BN, Lowe J, Vinters H. Parasitic infections. In: Neuropathology: a reference text of CNS pathology. 2nd ed. Philadelphia, Pa: Mosby, 2004; 379-81.

21. Tuzun M, Hekimoglu B. Hydatid disease of the CNS: imaging features. AJR Am J Roentgenol 1998; 171: 1497500.

22. Ciurea AV, Vasilescu G, Nuteanu L, Carp N. Cerebral hydatid cyst in children. Experience of 27 cases. Childs Nerv Syst 1995; 11: 679-86.

23. Shakeri M, Vahedi P. Cerebral Echinococcosis in the Children and Young Adults. Neurosurg Q 2007; 17: 2414.

24. Carrea R, Dowling E Jr, Guevara JA. Surgical treatment of hydatid cysts of the central nervous system in the pediatric age (Dowling's technique). Childs Brain 1975; 1: 4-21.

25. Kalaitzoglou I, Drevelengas A, Petridis A, Palladas P. Albendazole treatment of cerebral hydatid disease: evaluation of results with CT and MRI. Neuroradiology 1998; 40: 36-9.

26. Todorov T, Melchov G, Vutova K, Georgiev P, Lazarova I, Tonchev Z, et al. Factors Influencing the response to chemotherapy in human cystic echinococcosis. Bull WHO 1992; 70: 347-58.

27. Morris DL, Dykes PW, Marriner S, Bogan J, Burrows F, Skeene-Smith $\mathrm{H}$ et al. Albendazole-objective evidence of response in human hydatid disease. JAMA 1985; 253: 2053-7.

28. Tuzun M, Altinors N, Arda IS, Hekimoglu B. Cerebral hydatid disease CT and MR findings. Clin Imaging 2002; 26: 353-7.

29. El-Shamam O, Amer T, El-Atta MA. Magnetic resonance imaging of simple and infected hydatid cysts of the brain. Magn Reson Imaging 2001; 19: 965-74. 